\title{
Receptor-specific Bitterness Detection Using Immortal Cell Line and Impedance Sensor
}

\author{
Chunlian Qin ${ }^{1}$, Dongxiao Zhao ${ }^{2}$, Yuxiang Pan ${ }^{1}$, Zhen Qin ${ }^{1}$, Liquan Huang ${ }^{2, *}$, Ping Wang ${ }^{1, *}$ \\ ${ }^{1}$ Biosensor National Special Laboratory, Key Laboratory for Biomedical Engineering of Ministry of \\ Education, Department of Biomedical Engineering, Zhejiang University, Hangzhou 310027, China, \\ ${ }^{2}$ College of Life Sciences, Zhejiang University, Hangzhou 310013, China \\ Corresponding author's e-mail address: huangliquan@zju.edu.cn, cnpwang@zju.edu.cn
}

\begin{abstract}
:
Bitterness detection draws great attention in pharmaceutical and food industry. Existing detection methods relying on redox reactions lack specificity and most cell-based biosensors stay merely on cellular level thus ignore the specific receptor being activated. In this study, an impedance sensor is built to detect bitterness receptor-specifically using Caco-2 cell line, which expresses human T2R38 endogenously. After parameter optimization, this sensing system generates dose-dependent responses to known T2R38 ligands, phenylthiocarbamide and propylthioutacil. Quantitative study shows that the limits of detection are $0.094 \mu \mathrm{M}$ and $0.84 \mu \mathrm{M}$ respectively. Afterwards, specificity is also checked by other taste qualities and bitter compounds which cannot activate T2R38. In conclusion, this receptor-specific biosensor provides new platform for not only bitterness detection but also ligands detection, which is important in study such as mechanism of bitter perception and bitter masking.
\end{abstract}

Key words: Bitterness detection, Caco-2 cells, human T2R38, impedance sensor, receptor-specific

\section{Introduction}

Bitterness detection has attracted extensive attention in pharmacological and food safety issues. Traditional taste sensors using electrochemical sensor arrays are defective in specificity and fail to mimic the biological system. Other bioinspired taste sensor using mouse germ cell or rat gustatory system ignored the differences in bitter perception across species. More importantly, current cellbased taste sensors focus on cellular level, while neglect the binding releationship between bitter compounds and specific receptors[1]. In this study, a receptor-specific taste sensor is built to detect bitterness using Caco-2 cell line, which endogenously expresses human T2R38. Both qualitative and quantitative study has been done to check the specificity and sensitivity. What's more, bitter compounds activating other T2Rs rather than T2R38 are also tested. All the details are discussed in the following sections.

\section{Methods}

Caco-2 cell is seeded in 16-well Lab-tec chambers of impedance sensor and cultured in a standard humidified incubator.Chemical stimulation, including phenylthiocarbamide (PTC, $1 \mu \mathrm{M}-2 \mathrm{mM}$ ), propylthioutacil (Prop, $1 \mu \mathrm{M}$ -
$2 \mathrm{mM})$, quinine $(1 \mathrm{mM})$, denatonium benzoate $(1 \mathrm{mM})$, sucrose $(0.1 \mathrm{M}), \quad \mathrm{HCl} \quad(3 \mathrm{mM})$, monosodium glutamate $(3 \mathrm{mM}), \mathrm{NaCl}(0.1 \mathrm{M})$. As for data analysis, cell index $(\mathrm{Cl})$ is defined as:

$$
C I=\frac{Z_{\text {cell }}(f)}{Z_{\text {baseline }}(f)}-1
$$

where $Z_{\text {cell }}(f)$ and $Z_{\text {baseline }}(f)$ are the frequency-dependent electrode impedance with or without cells respectively. Normalization is performed to reduce the baseline difference across channels.

\section{Results and Discussion}

After being seeded on the sensor, two periods are observed chronologically, the attaching and proliferation period respectively (Fig.1a). Stable attachment forms after $5 \mathrm{~h}$, since the changing rates of $\mathrm{Cl}$ reach steady above zero (Fig.1b). Fig.1c shows cells on the electrodes of impedance sensor. Densities from $5 \mathrm{k}$ to $80 \mathrm{k}$ per well are checked to optimize the performance. $\mathrm{Cl}$ value shows that cells of $40 \mathrm{k}$ per well can generate best output in terms of area under curve (AUC) (Fig.2a). Micrographs also demonstrate that $40 \mathrm{k}$ cells are enough to fill up the sensor surface (Fig.2b). 
Quantitative study is performed using known ligands of T2R38, PTC and Prop (Fig.3). Dosedependent AUCs are observed and the limits of detection are calculated, $0.094 \mu \mathrm{M}$ and $0.84 \mu \mathrm{M}$ respectively. Compared with data in BitterDB $(0.04 \mu \mathrm{M}$ and $0.11 \mu \mathrm{M})$, which are obtained by biological methods[2], similar sensitivity is guaranteed.
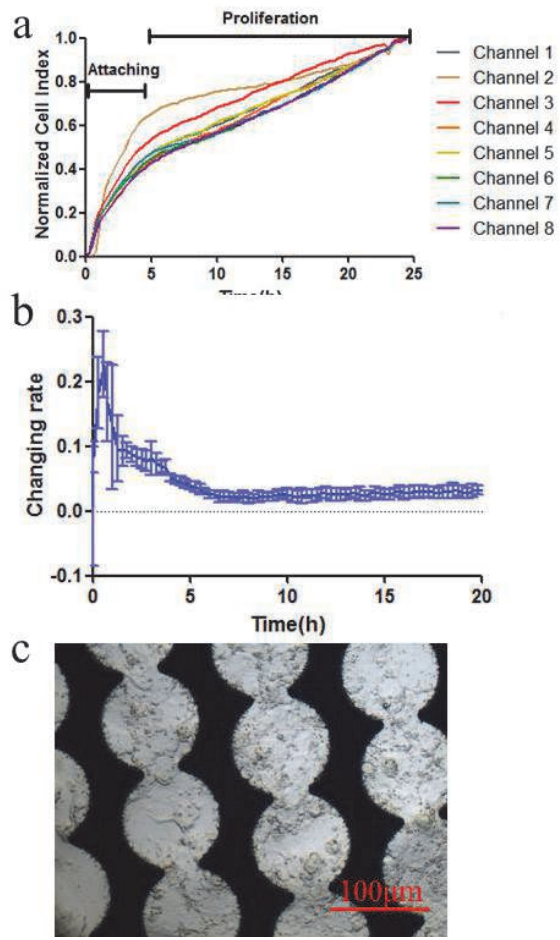

Fig. 1. (a) Two periods after seeding; (b) Changing rates of Cl; (c) Cells on the electrodes of sensor.

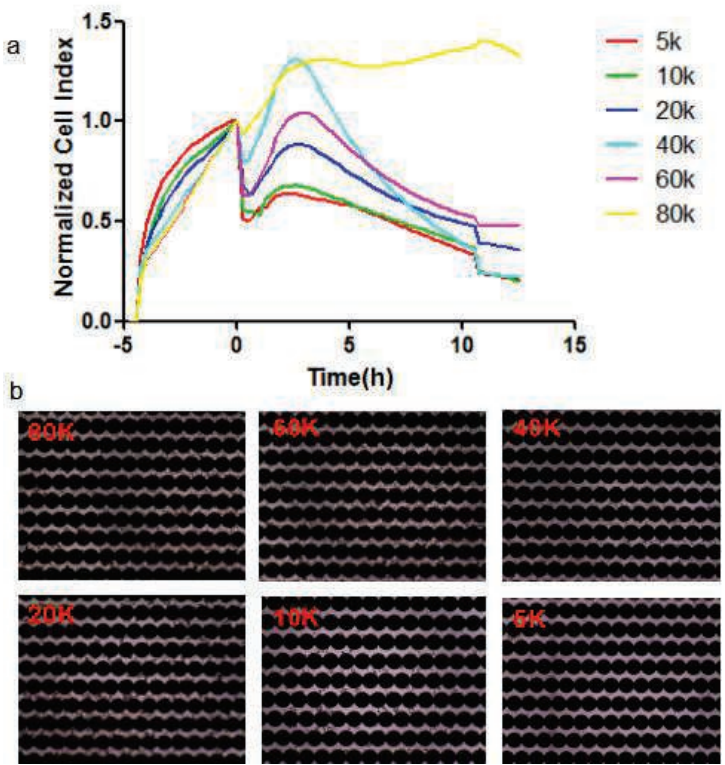

Fig. 2. (a) Densities affect magnitudes of responses (b) Micrographs of cells from $5 \mathrm{k}$ to $80 \mathrm{k}$ per well.

Specificity is tested first by other taste qualities, results show that hardly any responses are induced by sweetness, salt, sourness and umami (Fig.4). Afterwards, quinine (known to activates T2R4, 7, 10, 14, 39, 40, 43, 44, 46) and denatonium benzoate (known to activate T2R4, 8, 10, 13, 39, 43, 46, 47) are tested, and no significant responses are observed (Fig.5).

\section{Conclusion}

In this study, a receptor-specific sensor is developed to detect bitter compounds which specifically activates human T2R38. The cellbased biosensor can mimic the sensitivity of human bitter perception and avoids the interference of other taste qualities and other bitter compounds. This biosensor is promising in the research of ligands docking and mechanism of bitter taste.
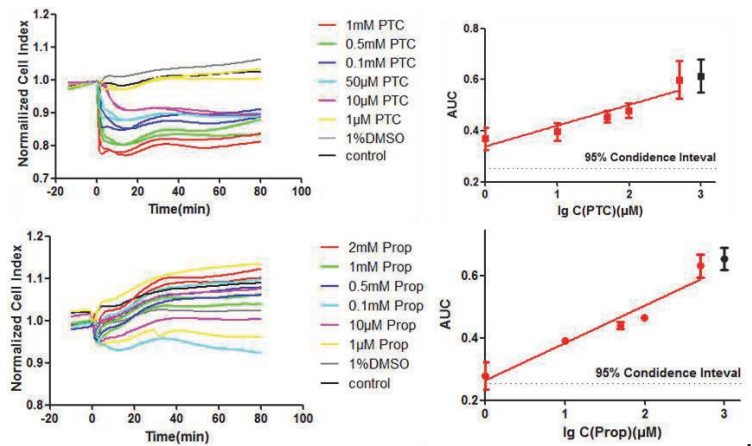

Fig. 3. Dose-dependent responses to PTC and Prop.

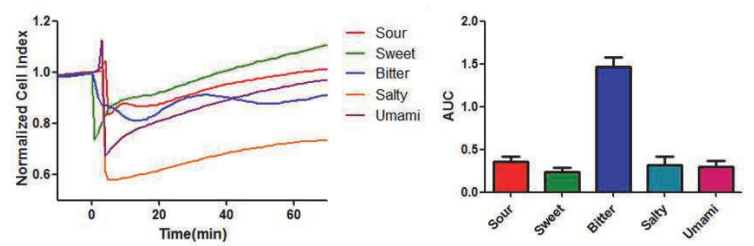

Fig. 4. Specificity to other taste qualities.

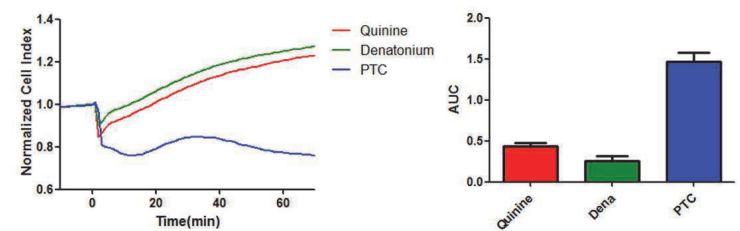

Fig. 5. Specificity to other bitter compounds.

\section{Acknowledgement}

This work was supported by the National Natur al Science Foundation of China (Grant No. 316 61143030)

\section{References}

[1] Q. Liu, C. Wu, H. Cai, N. Hu, J. Zhou, P. Wang Cell-based biosensors and their application in biomedicine. Chemical reviews. 114(12), 64236461(2014); doi: 10.1021/cr2003129

[2] W. Meyerhof, C. Batram, C. Kuhn, A. Brockhoff, E. Chudoba, B. Bufe, G. Appendino, M. Behrens. The molecular receptive ranges of human TAS2R bitter taste receptors, Chemical senses. 35(2), 157-170(2010); doi: 10.1093/chemse/bjp092 